\title{
Nivolumab-Induced Autoimmune Haemolytic Anaemia and Safety of Subsequent Use of Ipilimumab: A Case Report
}

\author{
Anna Carbó-Baguéa, boser Fort-Culillas ${ }^{a}$ Helena Pla-Juher ${ }^{a}$ \\ Jordi Rubió-Casadevalla, b \\ ${ }^{a}$ Catalan Institute of Oncology, Medical Oncology Department, Girona, Spain; biomedical \\ Research Institute (IDIBGI), Descriptive Epidemiology, Genetics and Cancer Prevention \\ Group, Girona, Spain
}

\section{Keywords}

Immune checkpoint inhibitors - Immune-related adverse event - Autoimmune haemolytic anaemia $\cdot$ Nivolumab $\cdot$ Ipilimumab

\begin{abstract}
Autoimmune haemolytic anaemia (AIHA) is a rare immune-related adverse event and appears to be more common with anti-PD1/PDL1 than anti-CTLA4. Little is known about the safety of re-treating with anti-PD1/PDL1 or changing to anti-CTLA4. We present a case of grade 4 AlHA due to nivolumab (PD1-inhibitor) treatment in a patient with melanoma for adjuvant setting after surgery and the safeness of subsequent treatment with ipilimumab (anti-CTLA4). After the remission of AlHA with steroids, ipilimumab was started with the rationale of its different mechanism of action. Fortunately, AlHA did not recur. The mechanism by which checkpoint inhibitors cause AIHA is likely by augmenting or redirecting immune surveillance, especially by activating pre-existing red blood cell autoantibodies, but further studies must be done. To our knowledge, this is the first case published in the literature with the change of immunotherapy treatment to anti-CTLA4.
\end{abstract}

\section{Karger ${ }^{\prime \prime}=$}




\section{Introduction/Background}

Immunotherapy has been an emerging treatment since 2015, and it is currently being used to treat many types of cancer with particular side effects different from those of chemotherapy. Tumours can evade normal immune surveillance by several mechanisms including upregulation of immune checkpoint molecules such as PD1 and PD ligand 1 or CTLA4.

Nivolumab is a fully human IgG4 monoclonal antibody which binds to and blocks the activation of PD1 as a checkpoint inhibitor (CPI). This release of check on the immune system can also trigger a reaction against the body's own tissues leading to autoimmune adverse effects such as pneumonitis, hepatitis, colitis, hypophysitis, arthritis, or nephritis, which are the most known side effects that appear in between 20 and $30 \%$ of the patients [1].

Autoimmune haemolytic anaemia (AIHA) has been described as a very uncommon immune-related adverse effect. We present a case of AIHA in a patient treated with nivolumab

for adjuvant setting after melanoma surgery and treated later with ipilimumab, a fully human IgG1k against CTLA4, without reproducing this type of toxicity.

\section{Case Report}

A 62-year-old male was diagnosed with BRAF-negative stage IVa completely excised acral melanoma in February 2019. He was considered for adjuvant nivolumab $3 \mathrm{mg} / \mathrm{kg}$ every 2 weeks [2]. In June 2019, after the third cycle/dose, he presented to the emergency room with severe asthenia and fatigue. He claimed not to have shortness of breath, thoracic pain, fever, or bleeding episodes. Physical examination showed mild conjunctival jaundice. The rest of the clinical examination was unremarkable. Routine laboratory tests showed $5.8 \mathrm{~g} / \mathrm{dL}$ haemoglobin levels, 1,200 absolute neutrophil count, indirect hyperbilirubinaemia $2.4 \mathrm{mg} /$ dL, high lactate dehydrogenase (LDH) $912 \mathrm{U} / \mathrm{L}$, and low haptoglobin $<10$ (Fig. 1-3). The direct antiglobulin test was positive for complement $3 \mathrm{~d}$ but negative for IgM and IgG.

Our diagnosis was AIHA. Considering that the patient was currently on immunotherapy treatment and given the timing association between nivolumab and anaemia, we could establish the immune-related adverse event grade 4 . The patient had not started other concomitant medications associated with AIHA, so we concluded it could be reasonably related to nivolumab.

We started treatment with a high dose of methylprednisolone $(1 \mathrm{mg} / \mathrm{kg})$ and 3 red blood cell transfusions. After 4 days, the haemoglobin levels raised to $9.5 \mathrm{~g} / \mathrm{dL}$, the bilirubin levels became normal, and LDH levels took a bit longer to normalize (Fig. 1-3). The patient was feeling well, so he was discharged from the hospital with a slow descending dose of cortisone. We decided to stop adjuvant treatment and start controls.

The first recurrence was detected after 1 year. He came with skin metastases, and CT scans showed one unique 6-mm temporal cerebral lesion. He underwent radiosurgery with complete response. Despite having suffered a CTCAE grade 4 immuno-related event, we thought to give a chance with ipilimumab (anti-CTLA4) as it was another mechanism of action. The first cycle was given at $1 \mathrm{mg} / \mathrm{kg}$ and next at $3 \mathrm{mg} / \mathrm{kg}$. No immune-related adverse events were found during the treatment. Four weeks after the fourth cycle of ipilimumab, abdominal adenopathies and new skin metastases appeared.

In August 2020, he started second line with fotemustine $80 \mathrm{mg} / \mathrm{m}^{2}$ every 2 weeks. After 7 cycles, a PET scan was performed with partial response only, persisting one skin

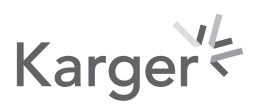


Case Reports in Oncology
Case Rep Oncol 2021;14:1289-1294

DOI: $10.1159 / 000518530$

(c) 2021 The Author(s). Published by S. Karger AG, Basel www.karger.com/cro

Carbó-Bagué et al.: Nivolumab-Induced Autoimmune Haemolytic Anaemia and Subsequent Use of Ipilimumab
Fig. 1. Evolution of lactate dehydrogenase levels.

Fig. 2. Evolution of bilirubin levels.

Fig. 3. Evolution of haemoglobin levels.

\section{Lactate dehydrogenase}

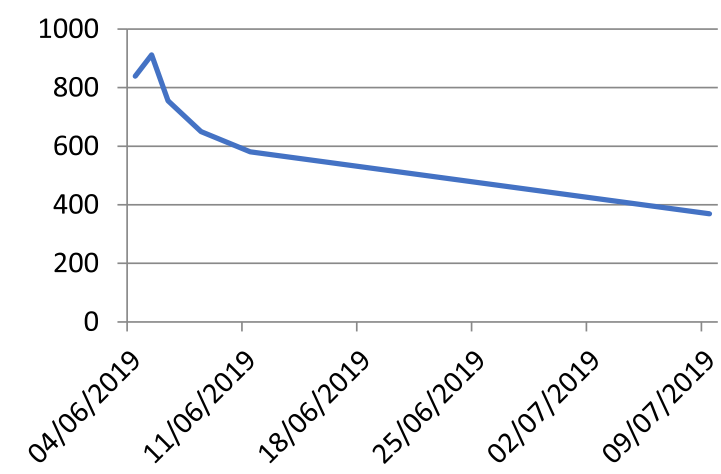

\section{Bilirubin}

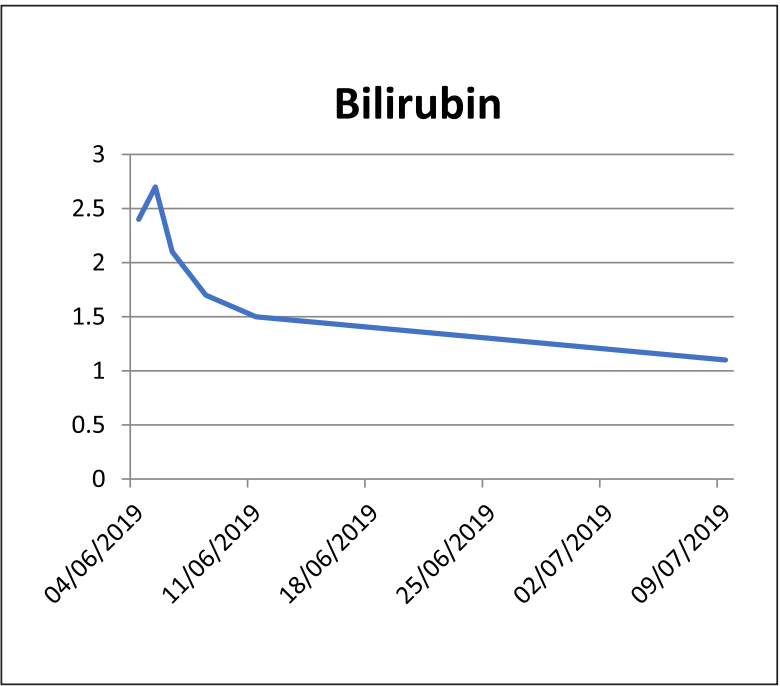

$0 x$

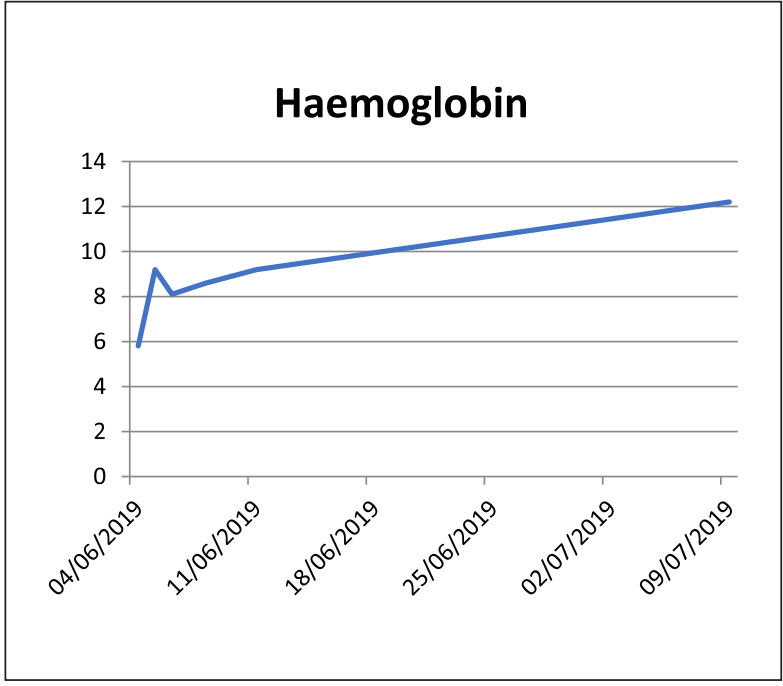


metastasis in the pretibial zone. In December 2020, he received surgery of the unique active focus on the skin with free margins. He continued with fotemustine every 3 weeks for better tolerance. In the last follow-up in April 2021, PET scan showed complete metabolic response.

\section{Discussion}

Novel immunotherapies such as anti-PD1 and anti-PDL1 can induce immune-related adverse events (irAEs) that can involve all organs. Haematological irAEs are rare and not extensively described in the literature.

A recent observational study by Delanoy et al. [3] found that the frequency of haematological irAEs associated with anti-PD1 and anti-PDL1 is low, mostly appearing as neutropenia, haemolytic anaemia, and thrombocytopaenia. Although grade 2 or worse have been described in $<1 \%$, those were serious and/or life threatening.

There are few case reports in the literature, 10 with nivolumab and 3 with pembrolizumab. We have performed a review of the literature and compared our case report to others published since the moment about solid tumours.

In the largest review published, a total of 68 cases were identified in the database of the Food and Drug Administration (FDA), 43 were associated with nivolumab, 13 with pembrolizumab, 7 with ipilimumab, and 5 with atezolizumab. Cases were included until March 2018, but a few of them have been published. Thus, AIHA is a rare but potentially serious adverse effect. Although the precise frequency is unclear, the estimated incidence of AIHA induced by CPI is $<0.1 \%$. It seems to be more common with anti-PD1 and anti-PDL1 than with anti-CTLA4, and apparently its frequency is not related to the underlying malignancy [4].

Drug-induced AIHA may be either "warm" or "cold" depending on the temperature at which the autoantibodies become active. Warm AIHA is generally mediated via IgG or via $\mathrm{C} 3$, and the autoantibodies are active at temperatures $>37^{\circ} \mathrm{C}$. Cold AIHA is mediated via IgM activation, and the autoantibodies are active at temperatures of $0-4^{\circ} \mathrm{C}$. AIHA after nivolumab therapy appears to be warm and is commonly mediated through IgG or C3 which was our case, but there are others published with IgG positive alone or both [5].

In addition, Michot et al. [6] found bone marrow failure in 3 cases of nivolumab therapy for melanoma, suggesting a central origin for immune-related cytopaenia as well. We do not think that this is our case, but physicians should suspect bone marrow suppression in some cases when pancytopaenia appears because AIHA has been described together with bone marrow aplasia [7]. Then, a bone marrow biopsy needs to be done.

The diagnosis of AIHA is made through laboratory findings. In our case, a decrease in haemoglobin, indirect hyperbilirubinemia, elevated LDH, and reduced haptoglobin were found. Coomb's test was positive for complement $3 \mathrm{~d}$ but negative for IgG, according to most of the cases reported in the literature $[3,4]$.

Clinical guidelines recommend corticosteroids as the treatment of choice in AIHA. In refractory cases, rituximab with or without intravenous immunoglobulins may be useful $[3,4]$.

First-line treatment choice was high-dose prednisolone, generally $1 \mathrm{mg} / \mathrm{kg}$, which required to be increased up to $1.5-2 \mathrm{mg} / \mathrm{kg}$ if the response is insufficient. Starting with high-dose pulse methylprednisolone $(1,000 \mathrm{mg} / 24 \mathrm{~h}$ per 3 days) has no evidence of better outcomes [3]. Treatment with rituximab associated with corticosteroids has also been reported [3]. Generally, anaemia responded fairly well to steroids, but it could be fatal, as in the cases described by Palla et al. [8], where despite increasing the dose of steroids, the 
patient expired due to respiratory failure, and Tanios et al. [4], where the patient developed bradycardia and cardiac arrest [9].

AIHA can occur at any time with nivolumab, so clinicians should be aware of it even if the patient has not suffered any haematologic events previously [8]. The median time between initiation of anti-PD1 or anti-PDL1 and occurrence of haem-irAE is 10 weeks, with a range between 2 and 78 weeks $[3,4]$. Our patient developed AIHA 7 weeks after the immunotherapy initiation, according to previous cases reported.

For patients with a history of mild AIHA diagnosis or a positive Coombs test without haemolysis, CPIs could probably be considered under special precautions and monitoring [4] assuming that in some cases, oral corticosteroids may be needed for recurrence especially if detected early by haemoglobin decrease in the routine blood tests as these patients should be monitored as a precaution [7].

After the remission of the AIHA with steroids, we started treatment with ipilimumab, an anti-CTLA4, based on the proven efficacy of this CPI in metastatic melanoma and due to its different mechanism of action compared to nivolumab. AIHA did not recur, and these data may provide some support for the safety of CPI in patients who have had haematological toxicities after anti-PD1/PDL1.

Schwab etal. [10] re-challenged a patient with ipilimumab and nivolumab, and the patient redeveloped AIHA. Conversely, Tardy et al. [11] re-challenged the patient with nivolumab without recurrence of AIHA.

The mechanism by which CPIs cause AIHA is likely by augmenting or redirecting immune surveillance, especially by activating pre-existing red blood cell autoantibodies. This mechanism of action is different from other drug-induced AIHA. It is speculated that CPIs cause a random activation of the immune system resulting in the formation of autoantibodies, activation of T-cell clones, and diminishing the function of regulatory $\mathrm{T}$ cells [12].

\section{Conclusions}

Haematological irAEs induced by anti-PD1 or anti-PDL1 are rare and appear to be more common with anti-PD1/PDL1 than anti-CTLA4. The estimated frequency of AIHA is $<1 \%$ of all immune-related side effects, but physicians should be aware because it often appears in a severe form.

Indications of re-challenge with immunotherapy remain unclear. It could be reasonable using anti-CTLA4 such as ipilimumab after a haematological irAE associated with anti-PD1/ PDL1 in metastatic melanoma, and our case report supports its safety. Ipilimumab is not free from AIHA risk but it is lower.

\section{Statement of Ethics}

Written informed consent was obtained from the patient for publication of this case report. The study is exempt from ethics committee approval because there is no experimental intervention, and we only describe a case report about routine and standard clinical practice. Patient's identity has been protected and treated confidentially. 


\section{Case Reports in Oncology}

Case Rep Oncol 2021;14:1289-1294

\begin{tabular}{l|c|c|}
\hline DOI: $10.1159 / 000518530$ & $\odot 2021$ The Author(s). Published by S. Karger AG, Basel
\end{tabular} www.karger.com/cro

Carbó-Bagué et al.: Nivolumab-Induced Autoimmune Haemolytic Anaemia and Subsequent Use of Ipilimumab

\section{Conflicts of Interest Statement}

The authors have no conflicts of interest to declare.

\section{Funding Sources}

No funding was received for this study.

\section{Author Contributions}

All authors helped to draft the manuscript, read, and approved the final manuscript. A.C. and R.F. are co-authors and were involved in collecting the data, performing the analysis, and writing the manuscript. H.P. participated in the analysis of the data. J.R. coordinated the drafting of the manuscript and its preparation for publication.

\section{Data Availability Statement}

All data generated or analysed during this study are included in this article and/or its online suppl. material files (for all online suppl. material, see www.karger.com/doi/10.1159/ 000518530). Further enquiries can be directed to the corresponding author.

\section{References}

1 Naidoo J, Page DB, Li BT, Connell LC, Schindler K, Lacouture ME, et al. Toxicities of the anti-PD-1 and anti-PDL1 immune checkpoint antibodies. Ann Oncol. 2015;26:2375.

2 Weber J, Mandala M, Del Vecchio M, Gogas HJ, Arance AM, Cowey CL, et al. Adjuvant nivolumab versus ipilimumab in resected stage III or IV melanoma. N Engl J Med. 2017;377:1824-35.

3 Delanoy N, Michot JM, Comont T, Kramkimel N, Lazarovici J, Dupont R, et al. Haematological immune-related adverse events induced by anti-PD-1 or anti-PD-L1 immunotherapy: a descriptive observational study. Lancet Haematol. 2019;6(1):e48-57.

4 Tanios GE, Doley PB. Autoimmune hemolytic anemia associated with the use of immune checkpoint inhibitors for cancer: 68 cases from the food and drug administration data base and review. Eur J Haematol. 2019;102: 157-62.

5 Palla AR, Khimani F, Craig MD. Warm autoimmune hemolytic anemia with a direct antiglobulin test positive for C3 and negative for IgG: a case study and analytical literature review of incidence and severity. Clin Med Insights Case Rep. 2013;6:57-60.

6 Michot JM, Vargaftig J, Leduc C, Quere G, Burroni B, Lazarovici J, et al. Immune-related bone marrow failure following anti-PD1 therapy. Eur J Cancer. 2017 Jul;80:1-4.

7 Ni D, Smylie M, Michael Smyliec. AIHA and pancytopenia as complications of pembrolizumab therapy for metastatic melanoma: a case report. Case Rep Oncol. 2019 May-Aug;12(2):456-65.

8 Palla AR, Kennedy D, Mosharraf H, Doll D. Autoimmune hemolytic anemia as a complication of nivolumab therapy. Case Rep Oncol. 2016;9:691-7.

9 Khan U, Ali F, Khurram MS, Zaka A, Hadid T. Immunotherapy-associated autoimmune hemolytic anemia. J Immunother Cancer. 2017;5:15.

10 Schwab KS, Heine A, Weimann T, Kristiansen G, Brossart P. Development of hemolytic anemia in a nivolumabtreated patient with refractory metastatic squamous cell skin cancer and chronic lymphatic leukemia. Case Rep Oncol. 2016;9:373-8.

11 Tardy MP, Gastaud L, Boscagli A, Peyrade F, Gallamini A, Thyss A. Autoimmune hemolytic anemia after nivolumab treatment in hodgkin lymphoma responsive to immunosuppressive treatment. A case report. Hematol Oncol. 2017 Dec;35(4):875-7.

12 Ogawa K, Ito J, Fujimoto T, et al. Exacerbation of autoimmune hemolytic anemia induced by the first dose of programmed death-1 inhibitor pembrolizumab: a case report. Invest New Drugs. 2018;36:509-12. 